\title{
O Fator de Impacto e as boas práticas de avaliação científica
}

\author{
Journal Impact factor and good scientific assessment practices
}

\author{
Catia Candida Almeida \\ Doutora em Ciência da Informação \\ Universidade Estadual Paulista \\ caticandida@gmail.com
}

Maria Claúdia Cabrini Gracio

Doutora em Letras

Doutora em Ciência da Informação

cabrini.gracio@unesp.br

\section{Resumo}

O objetivo desse estudo é apresentar os princípios norteadores de boas práticas de avaliação científica evidenciando a presença do indicador Fator de Impacto no contexto da avaliação científica. Adotou um levantamento bibliográfico de documentos que sugerem boas práticas de avaliação científica, a saber: DORA, Manifesto de Leiden e do Comitê de Avaliação Internacional de Pesquisa Quantitativa de Matemática e Estatística. Realizou-se uma análise comparativa dos conteúdos desses três manifestos, apontando as principais recomendações de boas práticas de avaliação científica. Seguindo essa perspectiva, o estudo mostra os princípios regidos pela CAPES à luz das recomendações internacionais de boas práticas de avaliação científica. Aponta-se que o indicador Fator de Impacto está presente no contexto das avaliações científicas e os documentos recomendam a não utilização desse indicador ou escolha de novas métricas e indicadores que sucedem o Fator de Impacto. Entretanto, contrariando as recomendações internacionais o Fator de Impacto é um dos indicadores mais utilizados nas avaliações científicas no Brasil. Desse modo, ressalta-se a avaliação da produção científica brasileira não deve ser sustentada por único indicador, corre-se o risco de levar a tomada de decisões incorreta e inconsistentes.

\section{Palavras-chave}

Avaliação científica. Boas práticas de avaliação científica. Ciência da Informação. Fator de Impacto.

\section{Abstract}

The objective of this research is to present the principles of good scientific evaluation practices, showing the presence of the Impact Factor indicator in the context of scientific evaluation. It adopted a bibliographic survey of documents that suggest good scientific evaluation practices: DORA, Leiden Manifesto and the International Evaluation Committee for Quantitative Research in Mathematics and Statistics. A comparative analysis of the contents of these three manifestos was carried out main recommendations for good scientific evaluation practices. Following this perspective, the study shows the principles governed by CAPES in the light of international recommendations for good scientific evaluation practices. It is pointed out that the Impact Factor indicator is present in the context of scientific assessments and the documents recommend not using that indicator or choosing new metrics and indicators that follow the Impact Factor. However, contrary to international recommendations, the Impact Factor is one of the most used indicators in scientific assessments in Brazil. Thus, it is emphasized that the evaluation of Brazilian scientific production should not be supported by a single indicator, there is a risk of leading to incorrect and inconsistent decision-making.

\section{Keywords}

Scientific evaluation. Good practices of scientific evaluation. Information Science. Journal Impact Factor. 


\section{INTRODUÇÃO}

A avaliação do comportamento e desempenho científico de diferentes áreas e países é uma atividade que pode contribuir para a identificação de frentes de pesquisa, questões e temas contemporâneos entre pesquisadores ou áreas, subsidiando as políticas científicas e tomadas de decisões.

Nesse contexto de avaliação científica a Bibliometria desenvolve métodos quantitativos de mensuração da literatura, documentos e outros meios de comunicação a fim de medir o estado da ciência e a tecnologia científica, sendo uma forma de situar a produção de um país em relação ao mundo, uma instituição a um país e os cientistas às suas próprias comunidades (SPINAK, 1998; MACIAS-CHAPULA, 1998). A Bibliometria assume um papel importante no desenvolvimento de indicadores quantitativos, particularmente, em relação aos indicadores de citação ou indicadores de impacto de citação, com o propósito de mensurar a intensidade do impacto das publicações científicas, calculados a partir da contagem das citações recebidas. Entre os indicadores de citação destaca-se o Fator de Impacto (Journal Impact Factor) (SPINAK, 1998; WALTMAN, 2016).

O Fator de Impacto foi desenvolvido por Eugene Garfield, do Institute for Scientific Information (ISI), com o propósito de selecionar revistas científicas da base de dados Science Citation Index ( $\mathrm{SCl})$, a fim de dar visibilidade ao impacto das revistas com pequeno volume de artigos publicados, mas de grande relevância para o desenvolvimento da ciência (GARFIELD, 1955, 1972; GARFIELD; SHER, 1963). Esse indicador retrata a frequência com que em média um artigo foi citado em um periódico, sendo definido como o quociente entre o número de citações recebidas no ano atual relativo aos artigos publicados nos dois anos anteriores e o número total de documentos publicados nesses dois anos anteriores. Além disso, o Fator de Impacto é um dos componentes do Jornal Citation Report (JRC), fornece ferramentas quantitativas para classificar, avaliar, categorizar e comparar periódicos científicos (CLARIVATE ANALYTICS, 2018).

No entanto, diversas críticas e limitações têm sido apresentadas na literatura quanto a sua metodologia de construção e utilização na avaliação das pesquisas (FERNANDESLLIMÓS, 2003; GLÄNZEL; MOED, 2002; SEGLEN, 1997; SIMONS, 2008; WALTMAN, 2016).

Van Leeuwen (2012) resume as principais críticas relacionadas ao Fator de Impacto em dois âmbitos: aspecto matemático/numérico e aspecto metodológico/utilização.

O aspecto matemático/numérico é referente a formulação matemática do cálculo do Fator de Impacto que contabiliza a quantidade de citação e documentos. No cálculo existe uma desigualdade na contabilização de quantidade que envolvem as classificações dos tipos de documentos chamados de "itens citáveis" (artigos de pesquisa original ou de revisão) e os "itens não citáveis" (notas técnicas, editoriais, cartas, resumos de reuniões, entre outros documentos). $\mathrm{O}$ autor afirma que o ISI, atualmente Clarivate Analytics responsável pela base de dados da Web of Science, contabiliza a quantidade de citação considerando os documentos "itens não citáveis" e a quantidade de documentos considerando apenas os "itens citáveis", ocorrendo assim, uma desigualdade na contabilização de quantidade de citação e documentos, influenciando no cálculo do Fator de Impacto e gerando distorções nos valores de Fator de Impacto de muitos periódicos, uma vez que os periódicos têm diferentes idades de literaturas citadas e ritmos de obsolescências (ritmo de citação do documento ao longo dos anos).

O aspecto metodológico/utilização, Van Leeuwen (2012) considera os seguintes pontos: a) os valores de Fator de Impacto dos periódicos não são normalizados (padronizados) por área de conhecimento, tornando os valores incomparáveis, por exemplo, os valores de Fator de Impacto da área de Biomedicina tendem a superar os valores de Fator de Impacto 
da área de Ciências Sociais; b) Os valores de Fator de Impacto não são normalizados por composição de periódico em termos de tipo de documentos publicados, ou seja, os periódicos que contemplam mais artigos de revisão tendem a ter maiores valores de Fator de Impacto. Ainda, o problema da classificação dos documentos em artigo de revisão destinados a periódicos que publicam artigos de revisão, não é de forma clara, lembrando que é feita por algoritmos computacionais não aberto ao pesquisador ou interessados; c) Janela de citação, a fórmula de cálculo do fator de Impacto considera o período temporal de dois anos para contabilização da quantidade de citação e documento, apresentando desvantagens para áreas em que o impacto do documento citado ocorre após um período mais longo, como o caso das áreas de Ciências Sociais que difere da Biomedicina, o impacto do documento citado geralmente ocorre em média de um a dois anos.

Apesar das críticas apontadas na literatura, o Fator de Impacto passou a alcançar prestígio nas avaliações científicas. Inicialmente, o Fator de Impacto foi amplamente utilizado para apoiar decisões de compra de coleção de periódicos e na gestão das bibliotecas (DE BELLIS, 2009).

Na subsequência, o Fator de Impacto passou a ser utilizado para classificar a "importância das revistas", sendo um dos fatores de decisão para a escolha de um periódico para a publicação de artigos por parte dos autores e promoção dos periódicos pelas editoras (BORGES, 2016; DE BELLIS, 2009).

No período mais recente, o Fator de Impacto vem sendo utilizado de forma mais ampla como sinônimo da qualidade das revistas, em que os gestores da ciência começaram a empregá-lo como ferramenta de tomada de decisão em questões de política científica envolvendo pesquisadores, grupos de pesquisa, departamentos, instituições e países (DE BELLIS, 2009).

Todavia, tem-se observado que alguns países empregam esse indicador como estratégia de avanço e maior visibilidade científica. Como exemplo ilustrativo, cita-se o fato ocorrido nos Ministérios da Ciência da Coréia do Sul, China e Paquistão, que ofereceram recompensas em dinheiro para que seus cientistas publicassem artigos em periódicos de "alto valor de impacto", como Nature, Science e Cell, entre outros. (AL-AWQATI, 2007).

Por outro lado, as agências de financiamento de pesquisa desenvolvem mecanismos de avaliação da produção científica dos pesquisadores e instituições para a alocação de recursos financeiros. Nesse processo avaliativo, um exemplo para ilustrar aplicação do Fator de Impacto, o caso da área da Medicina na Alemanha, em que esse indicador é utilizado para a alocação e distribuição dos recursos financeiros entre os departamentos, apontando para um problema complexo, por conta da heterogeneidade das disciplinas, tais como, genética molecular, cirurgia, medicina dentária, informática e médica, medicina social e outras áreas (KALTENBORN, 2004).

Em contrapartida, as instituições educacionais e de pesquisa produzem rankings de classificação dos periódicos baseados no Fator de Impacto e o desempenho individual do pesquisador pode ser medido por suas publicações em periódicos com alto valores de Fator de Impacto, atribuindo a maior chance do candidato superar os outros candidatos na promoção de carreira, bolsa ou financiamento de projetos. Em alguns países europeus, é uma prática constante a associação do Fator de Impacto ao mérito individual do pesquisador (BREMBS, 2018; DE BELLIS, 2009; PENNER et al., 2013; VARIN; CATTELAN; FIRTH, 2016).

Contudo, a forma de utilização do Fator de Impacto no contexto brasileiro não é diferente dos outros países, em que além de avaliar periódicos, também avalia a qualidade dos artigos individuais e o desempenho acadêmico de pesquisadores em editais para obtenção de bolsas de pesquisas, alocação e financiamento de recursos destinados às pesquisas, práticas essas contestadas por vários pesquisadores. 
As instituições educacionais e de pesquisa brasileira que oferecem programas de pósgraduação, sendo que os processos avaliativos dos órgãos de acompanhamento e avaliação, destaca-se a Coordenação de Aperfeiçoamento de Pessoal de Nível Superior (CAPES). Um dos instrumentos utilizado na avaliação dos programas de pós-graduação é o sistema Qualisperiódicos que consiste na classificação de periódicos utilizando o Fator de Impacto como um dos critérios de avaliação da produção científica brasileira (BARATA, 2016; BRASIL, 2018; CAMPOS, 2010).

Pensando na problemática de avaliação científica em que está inserido o Fator de Impacto e o reconhecimento dos problemas que envolviam os indicadores e as métricas de avaliação científica, as comunidades científicas estimularam debates e manifestos sobre a utilização adequada dos indicadores de avaliação científica em diferentes contextos com o propósito de promover boas práticas de avaliação científica.

Neste sentido, destacam-se os documentos: Declaração de São Francisco, Declaration on Research Assessment (DORA), (DORA, 2012), o Manifesto de Leiden (HICKS et al., 2015) e a recomendação do Comitê de Avaliação Pesquisa Quantitativa juntamente com a União Internacional de Matemática, Conselho Internacional de Matemática Aplicada e Industrial e o Instituto de Matemática e Estatística (ADLER; EWING; TAYLOR, 2009). Além do posicionamento da CAPES diante da perspectiva dos manifestos internacionais.

Assim, este trabalho tem o objetivo de apresentar os princípios norteadores de boas práticas de avaliação científica, evidenciando a presença do Fator de Impacto neste contexto. Este trabalho justifica-se, sobretudo, a utilização constante do Fator de Impacto como um dos critérios de avaliação da produção científica brasileira. Em contrapartida, os documentos originados desses manifestos internacionais a favor de uma avaliação científica fundamentada em princípios de boas práticas científicas não recomendam a utilização do Fator de Impacto (DORA, 2012) ou sugerem formas de avaliação científica.

\section{METODOLOGIA}

Esta pesquisa é de natureza descritiva baseada em um levantamento de documentos referentes às boas práticas de avaliação científica. Desse modo, realizou-se um levantamento bibliográfico sobre o tema, escolheu-se primeiro a Declaração de São Francisco (DORA, 2012), abordando vários aspectos de avaliação científica e críticas direcionadas ao Fator de Impacto. Em seguida, o manifesto de Leiden (HICKS et al., 2015) discorre de maneira geral as boas práticas de avaliação científica e o uso de indicadores quantitativos e qualitativos apoiando as avaliações. O documento do Comitê de Avaliação Pesquisa Quantitativa juntamente com a União Internacional de Matemática, Conselho Internacional de Matemática Aplicada e Industrial e o Instituto de Matemática e Estatística (ADLER; EWING; TAYLOR, 2009), apresenta recomendações para os pesquisadores das áreas de Matemática e Estatística referentes as métricas e indicadores de avaliação científica, sendo que em muitos casos são criadas por pesquisadores de outras áreas sem levar em conta a perspectiva e o rigor da formulação matemática envolvida nos indicadores. Este documento pode evidenciar a maneira como os pesquisadores das áreas de exatas percebem os indicadores de avaliação científica expondo os pontos críticos e os pontos positivos das métricas e indicadores de avaliação científica. Além disso, mostrar que a preocupação com as boas práticas de avaliação científica é de todas as áreas científicas, inclusive da área de Matemática e Estatística.

Para análise desses documentos, inicialmente realizou-se uma leitura dos documentos. Em seguida, foi feito um resumo dos principais pontos que retratavam os aspectos de boas práticas de utilização adequada de indicadores de avaliação científica em cada um dos documentos. Para apresentar os principais pontos construiu-se um quadro mostrando os 
três manifestos com a finalidade de apontar a convergência ou divergência de ideias enfatizadas nos documentos.

Por fim, o propósito foi de expor as reflexões dos pesquisadores e agentes responsáveis pelo sistema de avaliação científica brasileira à luz desses manifestos internacionais (OLIVEIRA; AMARAL, 2017) e a expectativa de mudança do modelo de avaliação científica porvindouro a ser implantado conforme cenário atual da política direcionada a pósgraduação brasileira (BRASIL, 2019).

\section{RESULTADOS E DISCUSSÃO}

No âmbito de manifestações internacionais sobre a utilização adequada dos indicadores de avaliação científica, destacam-se os documentos: DORA, o Manifesto de Leiden e a recomendação do Comitê de Avaliação de Pesquisa Quantitativa juntamente com a União Internacional de Matemática, Conselho Internacional de Matemática Aplicada e Industrial e o Instituto de Matemática e Estatística.

A Declaração de DORA ocorreu em 2012, em São Francisco (EUA), durante a reunião anual da ASCB (American Society for Cell Biology). O documento foi assinado por 1.356 universidades e instituições e mais de 14.000 assinaturas individuais de professores, pesquisadores e indivíduos envolvidos com avaliação de pesquisa. Nessa declaração, o principal indicador mencionado foi o Fator de Impacto e as recomendações baseiam-se na eliminação de métricas relacionadas com esse indicador, incentivo à busca de novos indicadores e a avaliação de pesquisa considerando outras qualificações dos pesquisadores.

Um documento importante que apresenta recomendações de boas práticas de avaliação de desempenho de pesquisa é o Manifesto de Leiden, publicado em 2015, resultante das discussões ocorridas na Conferência Internacional de Indicadores em Ciência e Tecnologia (STI-2014), em Leiden. Esse documento contempla dez princípios e ressalta que as avaliações são cada vez mais direcionadas as métricas, destacando o uso do Fator de Impacto e menos em opiniões de especialistas (HICKS et al., 2015).

Outro documento elaborado por pesquisadores da área de Matemática e Estatística, Comitê de Avaliação Pesquisa Quantitativa juntamente com a União Internacional de Matemática, Conselho Internacional de Matemática Aplicada e Industrial e o Instituto de Matemática e Estatística, aponta as implicações e problemas de confiar totalmente em métricas de avaliações científicas e chama a atenção principalmente para a utilização do Fator de Impacto (ADLER; EWING; TAYLOR, 2009).

Os principais pontos dos três manifestos são descritos no Quadro 1.

Quadro 1 - Principais pontos DORA (2012), Hicks et al. (2015), Adler, Ewing e Taylor (2009)

(continua)

\begin{tabular}{|l|l|l|}
\hline \multicolumn{1}{|c|}{$\begin{array}{c}\text { Resumo dos principais pontos da } \\
\text { declaração (DORA, 2012) }\end{array}$} & $\begin{array}{c}\text { Resumo dos principais pontos do } \\
\text { Manifesto de Leiden (HICKS et al., } \\
\text { 2015) }\end{array}$ & $\begin{array}{l}\text { Resumos dos principais pontos da } \\
\text { declaração (ADLER; EWING; TAYLOR, } \\
\text { 2009) }\end{array}$ \\
\hline $\begin{array}{l}\text { (1) Não utilizar métricas baseadas no } \\
\text { Journal Impact Factor como medidas } \\
\text { de qualidade dos artigos, avaliação de } \\
\text { contribuições individuais do pesquisa- } \\
\text { dor ou em decisões de contratação, } \\
\text { promoção e financiamentos. }\end{array}$ & $\begin{array}{l}\text { (1) A avaliação quantitativa deve } \\
\text { apoiar a avaliação qualitativa conduzi- } \\
\text { da por especialista (facilitadora da } \\
\text { avaliação por pares). }\end{array}$ & $\begin{array}{l}\text { (1) A precisão das métricas é } \\
\text { A utilização indevida das estatísticas } \\
\text { de citações é ampla e conhecida, } \\
\text { apesar das várias advertências quanto } \\
\text { a esse tipo de uso. Entre esses usos, } \\
\text { mencionam-se as tomadas de decisão } \\
\text { fundamentadas em aplicações incor- } \\
\text { retas das estatísticas de citação, como } \\
\text { o Fator de Impacto, por governos, } \\
\text { instituições e pesquisadores. }\end{array}$ \\
\hline
\end{tabular}

Fonte: Elaboração própria (2019). 
Quadro 1 - Principais pontos DORA (2012), Hicks et al. (2015), Adler, Ewing e Taylor (2009)

(continuação/continua)

\section{Resumo dos principais pontos da declaração (DORA, 2012)}

(2) Explicação dos critérios adotados na avaliação da produtividade científica dos candidatos a financiamentos (direcionadas às agências de financiamento).

\section{Resumo dos principais pontos do Mani- festo de Leiden (HICKS et al., 2015)}

(2) Medição de desempenho dos indicadores em relação aos objetivos de pesquisa da instituição, grupo ou pesquisador. Os indicadores devem considerar contextos socioeconômicos e culturais. Um único modelo de avaliação não se aplica em todos os contextos.

(3) Consideração de várias medidas de impacto, incluindo indicadores qualitativos (direcionadas às agências de financiamento)
(3) Proteção da excelência em pesquisa relevante ao local, reconhecendo a excelência da pesquisa de local ou regional.
Resumos dos principais pontos da declaração (ADLER; EWING; TAYLOR, 2009)

(2) A confiança baseada exclusivamente nas métricas de citação nos julgamentos, em detrimento à revisão por pares. Aqueles que defendem a dependência das métricas baseadas em citações pressupõem que cada citação tem o mesmo significado e impacto.

(3) As estatísticas são valiosas para compreender o mundo e fornecem apenas uma compreensão parcial. No mundo contemporâneo, é necessário cuidado com as afirmações relativas à superioridade das medidas numéricas em relação às outras formas de compreensão e análise. Aqueles que defendem a utilização das estatísticas baseadas em citações podem ter uma compreensão incompleta da pesquisa. Neste sentido, é preciso usar as estatísticas de forma correta e com sabedoria.
(4) Esclarecimentos dos critérios utilizados nas decisões de contratação, permanência e promoção do pesquisador (direcionadas às instituições).

(5) Consideração de várias medidas de impacto, incluindo indicadores qualitativos (direcionadas às instituições).

(6) Redução do uso do Journal Impact Factor como uma ferramenta de avaliação, adotando outros tipos de métricas que podem fornecer uma visão apropriada do desempenho do periódico (direcionadas aos editores de periódicos).

(7) Disponibilização de métricas relativas ao artigo para promover a mudança de avaliação do conteúdo do artigo (direcionadas aos editores de periódicos).

(8) Promoção de práticas responsáveis de autoria e fornecimento de informações das contribuições específicas de cada autor (direcionadas aos editores de periódicos).
(4) Manutenção da obtenção de dados analíticos e abertos, transparentes e simples. A construção das bases de dados usadas para a avaliação da produção científica deve seguir regras predeterminadas e claras. Os avaliadores devem se esforçar na busca de equilíbrio entre o indicador simples e fidedigno à complexidade do processo de pesquisa.

(5) Possibilitar que avaliadores averiguem os dados e análises. Os envolvidos nesse processo devem assegurar a precisão dos dados, por meio de auto verificação ou de auditorias.

(6) Levar em consideração as variações das áreas nas publicações e práticas de citações. A melhor prática é selecionar um conjunto de indicadores para facilitar a escolha.

(7) Apoiar as avaliações de pesquisadores individuais em julgamentos qualitativos de seus próprios sistemas de avaliação. A abordagem deve considerar mais informações sobre o conhecimento, experiência, atividades e influência de um indivíduo.

(8) Evitar a objetividade mal empregada e a falsa precisão. Os indicadores de Ciência e Tecnologia são propensos a ambiguidades e incertezas conceituais. Quando identificados devem acompanhar os valores, e os produtores de indicadores devem evitar falsas precisões.

Fonte: Elaboração própria (2019). 
Quadro 1 - Principais pontos DORA (2012), Hicks et al. (2015), Adler, Ewing e Taylor (2009)

(continuação)

\begin{tabular}{|l|}
\hline $\begin{array}{c}\text { Resumo dos principais pontos da decla- } \\
\text { ração (DORA, 2012) }\end{array}$ \\
\hline $\begin{array}{l}\text { (9) Remoção das limitações de listas de } \\
\text { referência dos artigos nos periódicos de } \\
\text { acesso aberto, ou baseado em assinatu- } \\
\text { ra, e incentivar a disponibilização em } \\
\text { Creative Commons Public Domain Dedi- } \\
\text { cation (direcionadas aos editores de } \\
\text { periódicos). }\end{array}$ \\
\hline
\end{tabular}

(10) Redução das restrições dos números de referências de pesquisa quando apropriado, referenciar a literatura, a fim de dar crédito aos pesquisadores que relataram a descoberta (direcionadas aos editores de periódicos).

(11) Ser aberto e transparente, fornecendo dados e métodos usados para calcular as métricas (direcionadas às organizações que disponibilizam métricas).

(12) Disponibilização de dados, permitindo uma licença para reutilização irrestrita e o acesso computacional aos dados (direcionadas às organizações que disponibilizam métricas).

(13) Determinação da transparência, com medidas de combates à manipulação inadequada das métricas (direcionadas às organizações que disponibilizam métricas).

(14) Consideração dos diversos tipos de artigos e os diferentes campos e assuntos quando as métricas forem usadas (direcionadas às organizações que disponibilizam métricas).

(15) As decisões dos comitês de financiamentos, contratações e promoções devem ser baseadas em conteúdo científico em substituição das métricas (direcionadas aos pesquisadores).

(16) Quando for apropriado, referenciar a literatura primária, a fim de dar crédito ao responsável (direcionadas aos pesquisadores).

(17) Utilização de várias métricas e indicadores que evidenciam o impacto dos artigos (direcionadas aos pesquisadores).

(18) Incentivo e ensino de boas práticas de avaliação de pesquisa em oposição às práticas de avaliação que dependam do FI (direcionadas aos pesquisadores).

Fonte: Elaboração própria (2019).

\section{Resumo dos principais pontos do Manifesto de Leiden (HICKS et al., 2015)}

(9) Reconhecer o efeito sistêmico da avaliação e dos indicadores. Os indicadores podem ser modificados por meio diversos fatores, esses fatores precisam ser antecipados. Isso significa que um conjunto de indicadores é sempre preferível do que um único indicador para se tornar uma meta. (10) Examinar regularmente os indicadores e atualizá-los. Os objetivos das pesquisas, as avaliações e o próprio sistema de pesquisa mudam ou evoluem conjuntamente. Nesse cenário, as métricas tornam-se inadequadas, dando espaço para os novos indicadores. Os sistemas de indicadores devem ser revistos e modificados sempre que possível.

\section{Resumos dos principais pontos da declaração (ADLER; EWING; TAY- LOR, 2009)}


De acordo o documento (DORA, 2012), o Fator de Impacto assume o papel central nas discussões dos possíveis abusos cometidos nas avaliações científicas. Esse documento recomenda a não utilização do Fator de Impacto em classificações individuais de pesquisadores (em alguns países europeus o Fator de Impacto faz parte do processo avaliativo dos pesquisadores, como por exemplo, os pesquisadores que mais publicam trabalhos em revistas com o alto valor de Fator de Impacto, tendem a obter maiores pontuações nas avaliações individuais), em ambientes de avaliação de promoções de carreiras (destacam-se os pesquisadores que publicam em revista com alto valor de Fator de Impacto), bolsas de pesquisas (tendência dos pesquisadores que publicam em revistas com alto valor de Fator de Impacto conseguirem bolsas de pesquisa), financiamento de pesquisas, entre outras modalidades de avaliações.

Entretanto, suspender a utilização do indicador Fator de Impacto como um instrumento de avaliação, ainda é um desafio para as universidades, instituições e agências de financiamento de pesquisa. O relatório da European Commission (2017) de avaliação de carreiras dentro da prática de ciência aberta indica que algumas instituições signatárias do DORA não conseguiram implementar procedimentos de contratação e promoção de professores e pesquisadores que não decorram da utilização do Fator de Impacto como medida de avaliação individual. Esse relatório aponta que 14\% dos entrevistados das agências de financiamento de pesquisas signatárias do DORA, sendo $7 \%$ confirmaram que não implementaram procedimentos de avaliações independentes desse indicador.

Este fato reforça a ideia de que os argumentos de DORA não foram suficientes para diminuir a utilização do Fator de Impacto nas avaliações de pesquisa e principalmente, avaliação individual do pesquisador (EUROPEAN COMMISSION, 2017; MAISONNEUVE, 2015).

Por outro lado, no manifesto de Leiden é ressaltado que a avaliação quantitativa deve ser apoiada pela avaliação qualitativa conduzida por opinião de especialista, referindo a avaliação por pares. Isto indica pode indicar que uma avaliação científica não deve ser baseada apenas em indicadores ou métricas quantitativas. No caso de avaliações de pesquisadores recomenda-se incluir nos sistemas de avaliação julgamentos de aspectos qualitativos com informações de conhecimento, experiência, atividade e influência do indivíduo em sua área científica (HICKS et al., 2015).

Nessa perspectiva, o documento de DORA (2012) apresenta que as medidas ou indicadores de impacto devem incluir indicadores qualitativos direcionados às avaliações de candidatos (pesquisadores que pleiteiam promoção, bolsas e financiamentos de pesquisa) instituições e agências de financiamentos.

Entretanto, os documentos DORA (2012) e Hicks et al. (2015) não especificam o tipo de informação que contempla uma avaliação qualitativa, apenas evidenciam uma preocupação de que uma avaliação científica precisa considerar indicadores quantitativos juntamente com os qualitativos.

Para ter uma ideia de indicador qualitativo, o estudo de Almeida (2019) realizado com pesquisadores das áreas de Ciência da Informação e Matemática, Probabilidade e Estatística apontou uma sugestão de construção de um indicador qualitativo que assegure nas avaliações por pares a leitura completa do artigo e os aspectos que envolvem cada área científica.

Nota-se ainda, que os documentos DORA (2012) e Hicks et al. (2015) não apresentam uma sugestão específica de um indicador que configura boas práticas de avaliação científica. Os documentos apontam que os desenvolvedores de indicadores devem considerar o envolvimento dos contextos socioeconômicos e culturais e de aplicação do indicador (instituição, pesquisador, agência de financiamento), disponibilizar os métodos usados em seus cálculos, 
bem como, a base de dados, a fim demostrar transparência do processo. Os indicadores ou sistema de indicadores devem ser examinados regularmente e modificados sempre que possível e de forma clara e transparente para os pesquisadores. Além disso, é preciso levar em consideração que um único modelo de avaliação ou um único indicador não se aplica em todos os contextos de avaliação.

Adler, Ewing e Taylor. (2009) alertam para as métricas e indicadores de avaliação científica são fundamentadas em estatísticas incorretas de citações, como o Fator de Impacto, usado por governos, instituições e pesquisadores. Os autores ressaltam que muitas métricas científicas são baseadas em citações em detrimento da avaliação por pares. Para os que defendem apenas utilização das métricas baseadas em estatísticas de citações podem ter uma compreensão parcial ou incompleta da pesquisa.

DORA (2012) e Hicks et al. (2015) também fazem um alerta sobre as práticas de citações e a sua influência nas métricas e indicadores de avaliação científica.

Contudo, DORA (2012), Hicks et al. (2015), Adler, Ewing e Taylor (2009) evidenciam que, quando o Fator de Impacto é empregado na maioria das avaliações científicas de forma inadequada e pode prejudicar todos processos e procedimentos de avaliações, consequentemente, corre-se o risco de tomar decisões incorretas.

Em suma, os três documentos apresentam que as boas práticas de avaliação científica baseiam-se: desenvolvimento de indicadores que refletem os contextos de sua aplicação, os processos ou sistemas de avaliação precisam ter regras claras e transparentes para o pesquisador, os indicadores incluso no sistema de avaliação devem ser atualizados sempre que possível e tornar cada vez mais próximas ou conjuntas as avaliações quantitativas e qualitativas.

Waltman e Traag (2017) recomendam que as boas práticas em pesquisa decorrem dos argumentos contra o uso indiscriminado dos indicadores em avaliações, especialmente o Fator de Impacto. Os autores consideram que o Fator de Impacto não deve ser usado em avaliação de artigos individualmente, as instituições e agências de financiamento devem apoiar suas decisões em sistemas de avaliação que combinam informações qualitativas e quantitativas.

A ampla utilização e as limitações do Fator de Impacto e das bases de dados Web of Science levaram os pesquisadores a conduzirem projetos de melhoria do $\mathrm{Fl}$ e a desenvolver outras medidas e indicadores de impacto de citação como alternativa ou complementação (WALTMAN, 2016).

Hirst (1978) introduziu o Disciplinary Impact Factor (DIF), "Fator de Impacto de Disciplina", usado para classificar uma lista de periódicos dentro de uma mesma área e assunto científico. Baseado no número médio de vezes que um periódico foi citado em uma área.

Vinkler (1991) desenvolveu o Standard Journal Impact (SII), "Desvio do Impacto do Periódico", utilizado como um indicador de impacto padronizado para comparar os periódicos de diferentes áreas.

Van Leeuwen e Moed (2002) desenvolveram o indicador Journal to Field Impact Score (JFIS), "Impacto da Pontuação do Periódico na Área", pondera o impacto médio dos documentos de um periódico e o impacto médio de todos os documentos da área no período de classificação, por meio da normalização dos tipos de documentos e o ano de publicação.

Sombatsompop, Markpin e Premkamolnetr (2004) propuseram o Cited Half-Life Impact Factor (CHAL-IF), "Fator de Impacto de Meia-Vida de Citação", relaciona o número vezes em que o artigo foi citado no ano atual e o número de artigos publicados nos últimos anos, com o propósito de substituir o período de citação de dois anos pela meia-vida das citações de um periódico. 
Rousseau (2005) apresentou o indicador Median Impact Factor (MIF), "Fator de Impacto Mediano", definido como a metade do número total de citações recebidas no ano atual.

Leydesdorff e Bornmann (2011) introduziram o Integrated Impact Indicator (I3) "Indicador de Impacto Integrado" a fim de normalizar o impacto das citações, evitando que as análises sejam distorcidas em função das diferenças entre as áreas, quando se pretende fazer análises comparativas de diferentes áreas do conhecimento (GRACIO; OLIVEIRA, 2014). Esse indicador não é baseado apenas na contagem da média de citações e das publicações, mas usa a ordenação dos percentis das citações (por exemplo, superior a $1 \%$ superior a $10 \%$, etc.).

Nesse sentido, atualmente os pesquisadores e indivíduos envolvidos no processo de avaliação científica, estruturam grupo de pesquisa com propósito de elaborar indicadores e outras formas de avaliação científica baseadas na adoção de critérios transparentes para qualquer tipo de avaliação, sendo adequada para cada proposta de avaliação científica e também as partes envolvidas no processo de avaliação, compartilhando responsabilidades na construção e uso dos indicadores (WOUTERS, et al., 2019). Ademais, os três manifestos internacionais mencionados aqui provocaram reflexões nos pesquisadores e agentes responsáveis pelos sistemas de avaliações científicas no Brasil.

O sistema de avaliação da pós-graduação brasileira é orientado pela CAPES, responsável por incentivar as boas práticas na avaliação de desempenho de pesquisas. Os fundamentos dessa avaliação compreendem três principais pontos: (1i) qualidade assegurada pela análise dos pares; (2i) consulta e debate constante com a comunidade acadêmica na definição e atualização dos critérios de avaliação; (3i) transparência dos procedimentos e resultados (OLIVEIRA; AMARAL, 2017).

Nessa perspectiva, segundo Oliveira e Amaral (2017), os princípios regidos pela CAPES à luz dos manifestos internacionais:

\section{Quadro 2 - Principais pontos da avaliação da CAPES}

(1) Indicadores: importantes, mas não soberanos. Os indicadores são usados como ferramenta auxiliar à análise de mérito, não sendo o único e soberano parâmetro. A análise qualitativa de conteúdo é considerada fundamental para a avaliação e só é possível de ser feita pelos consultores.

(2) A importância da combinação de indicadores e da percepção das suas limitações. Os indicadores possuem vantagens e limitações, mas o mau uso acarreta distorções nas tomadas de decisão.

(3) Indicadores não são imutáveis. Os indicadores necessitam passar por atualizações periódicas e terem capacidade de adequação às mudanças.

(4) O limite da precisão. Os indicadores são representados por números, inteiros ou decimais, e devem ser interpretados com cautela.

(5) Transparência das ações. As formas de cálculos e resultados das avaliações precisam atender o princípio da transparência por qualquer interessado, sendo parte fundamental da isonomia do processo.

(6) Reconhecimento da diversidade entre áreas e o contexto do impacto dos programas. O impacto da pesquisa possui também abrangência diferenciada, que pode ser considerado em nível local, regional, nacional ou internacional. A CAPES estabelece os princípios gerais da avaliação, como a "ficha de avaliação" e seus quesitos e itens gerais que devem obrigatoriamente constar em todas as áreas.

(7) Medir a qualidade e o impacto da pesquisa quanto a transformar algo ao seu redor e contribuir com a solução de um problema da sociedade, preferencialmente, à quantidade.

(8) Bons indicadores necessitam de dados estruturados e confiáveis. O sistema de registro de dados precisa adotar padrões para estruturação e descrição da informação, mas necessita de ações para auditoria dos dados em bases internacionais e em outras fontes de informações.

Fonte: Elaboração com base em Oliveira e Amaral (2019). 
Nota-se que as recomendações apoiadas pela CAPES são importantes para o fortalecimento do sistema de avaliação da pós-graduação brasileira e necessitam de melhorias constantes em busca de uma ciência pautada em processos confiáveis.

No contexto de avaliação da produção científica, o sistema de avaliação da CAPES é aperfeiçoada de forma contínua para os programas de pós-graduação buscam padrões que representam a excelência acadêmica nacional (GUTIERRES; CABRINI, 2012).

Os indicadores assumem um papel relevante baseado na contagem bibliográfica e citações, entre eles, o Fator de Impacto usado nos procedimentos de avaliação da produção científica pela CAPES em várias áreas do conhecimento (VOGEL, 2017).

Entretanto, o Fator de Impacto deve ser utilizado com algumas ressalvas diante dos problemas metodológicos e de utilização que envolvem esse indicador (ALMEIDA, 2019).

Atualmente a CAPES, dedica-se ao aprimoramento e implantação de instrumentos relacionados a avaliação da pós-graduação brasileira. Um dos eixos temáticos é a proposta de abordagem de avaliação multidimensional dos programas de pós-graduação, usando o modelo criado pelo consórcio europeu U-Multirank (UMR) para classificar e ranquear as instituições de ensino superior de diversos países (BRASIL, 2019). Uma das diretrizes desse modelo é a inclusão de um conjunto de indicadores bibliométricos para classificação dos periódicos criados pela Clarivate Analytics e os campos de conhecimentos científicos, priorizando as áreas científicas que delineiam as coleções de periódicos da Web of Science (BRASIL, 2019).

O cenário aponta que o Fator de Impacto é um dos indicadores de citação que continuará a fazer parte dos instrumentos da avaliação da pós-graduação brasileira. Visto que, o Fator de Impacto é um dos principais indicadores da Clarivate Analytics, consequentemente fará parte do novo modelo de avaliação da CAPES.

Em contrapartida, as recomendações dos documentos (ADLER; EWING; TAYLOR, 2009; DORA, 2012; HICKS et al., 2015) condenam práticas de avaliação científica baseadas na utilização do Fator de Impacto.

\section{CONCLUSÃO}

Os indicadores bibliométricos têm sido utilizados amplamente em avaliações da produção científica, desempenhos de pesquisadores, universidades e países. Em especial, destaca-se o Fator de Impacto, um dos indicadores mais utilizados na avaliação da produção científica brasileira.

No contexto de avaliação científica, pesquisadores preocupados com a utilização de métricas e indicadores promoveram os manifestos internacionais que estimulam as boas práticas de avaliação científica, comumente conhecidas como: DORA, Manifesto de Leiden e do Comitê de Avaliação Pesquisas Quantitativas de Matemática e Estatística.

Esses documentos demonstraram o uso excessivo do Fator de Impacto em várias situações de avaliação científica, principalmente na avaliação individual do pesquisador. Os documentos recomendam que os gestores de avaliação científica e as agências fomentos e recursos financeiros de pesquisas recorram novas formas de avaliações, especialmente, com a combinação de indicadores quantitativos e qualitativos.

Entretanto, os três documentos apresentam considerações relevantes para o procedimento de avaliação de científica, mas não sugerem indicadores que configuram boas práticas de avaliação científica. Ressalta-se que existe na literatura vários indicadores que podem ser utilizados como uma alternativa ou complementação ao indicador Fator de Impacto.

Contrariando as recomendações internacionais à respeito das avaliações científicas, a CAPES, responsável pelas avaliações dos programas de pós-graduação brasileira, indica uma dependência do indicador Fator de Impacto nas avaliações de várias áreas científicas, de 
maneira direta, no quesito Produção Intelectual dos pesquisadores dos programas de pósgraduação, distribuição de recursos financeiros e bolsas de pesquisa acadêmica. Por meio do instrumento Qualis-Periódicos, sendo um sistema de classificação de produção científica dos programas de pós-graduação brasileira. Esse sistema contém critérios, regras e métricas quantitativas, em especial, o Fator de Impacto.

No cenário vindouro, com a implantação do modelo de avaliação da CAPES, indica que o Fator de Impacto continuará a ser utilizado nas avaliações científica brasileira, longe disso, poderá ficar apenas as iniciativas dos pesquisadores em desenvolver novas métricas, indicadores e formas de avaliação em consonância com as boas práticas internacionais de avaliação de pesquisa. Ainda, ressalta-se que a avaliação da produção científica não deve ser sustentada por um único indicador, corre-se o risco de levar a tomada de decisões incorreta e inconsistentes.

\section{REFERÊNCIAS}

ADLER, R.; EWING, J.; TAYLOR, P. Citation Statistics. Statistical Science, v. 24, n.1, p.1-14, 2009. Disponível em: https://projecteuclid.org/download/pdfview 1/euclid.ss/1255009002. Acesso em: 20 dez. 2019. doi: 10.1214/09-STS28.

AL-AWQATI, Q. Impact factors and prestige. Kidney International, v. 71, n. 3, p. 183-185, 2007. Disponível em:

https://www.researchgate.net/publication/6551721 Impact factors and prestige. Acesso em: 20 dez. 2019. doi:10.1038/sj.ki.5002094.

ALMEIDA, C. C. Fator de Impacto e avaliação da produção científica: compreensão na perspectiva das áreas de Ciência da Informação e Matemática, Probabilidade e Estatística. 2019. Tese (Doutorado em em Ciência da Informação) - Universidade Estadual Paulista Júlio de Mesquita Filho, Marília, SP. 2019. Disponível em:

https://repositorio.unesp.br/handle/11449/182447. Acesso em: 10 dez. 2019.

BARATA, R. C. B. Dez coisas que você deveria saber sobre o Qualis. RBPG, Brasília, v. 13, n. 30, p. 13 -40, jan./abr. 2016. Disponível em:

http://ojs.rbpg.capes.gov.br/index.php/rbpg/article/view/947. Acesso em: 20 nov. 2016.

BORGES, N. O fator de impacto e a avaliação da publicação científica. Revista Portuguesa de Cirurgia, Lisboa, n. 38, p.7-8, 2016. Disponível em:

http://www.scielo.mec.pt/scielo.php?script=sci arttext\&pid=S1646-69182016000500002. Acesso em: 15 janeiro 2018.

BRASIL. Coordenação de Aperfeiçoamento de Pessoal de Nível Superior (CAPES). História e missão. Brasília: CAPES, c2018. Disponível em: https://www.capes.gov.br/historia-e-missao. Acesso em: 25 nov. 2017.

BRASIL. Coordenação de Aperfeiçoamento de Pessoal de Nível Superior (CAPES). Relatório Técnico DAV Avaliação Multidimensional de Programas de Pós-Graduação. Brasília: CAPES, 2019. Disponível em:

https://www.capes.gov.br/images/novo portal/documentos/DAV/avaliacao/19072019 Rela torio-Tecnico-DAV-Avaliacao-Multidimensional-PPG.pdf. Acesso em: 02 out. 2019. 
BREMBS, B. Prestigious Science Journals Struggle to Reach Even Average Reliability. Frontiers Human Neuroscience, Switzerland, v. 12, p. 37, 2018. Disponível em: https://www.frontiersin.org/articles/10.3389/fnhum.2018.00037/full. Acesso em: 20 dez. 2019. doi: 0.3389/fnhum.2018.00037.

CAMPOS, J. N. B. Qualis periódicos: conceitos e praticas nas Engenharias I. RBPG, Brasília, v. 7, n. 14, p. 477-503, dez. 2010. Disponível em:

https://ojs.rbpg.capes.gov.br/Índex.php/rbpg/article/download/14/11. Acesso em: 22 mar. 2018.

CLARIVATE ANALYTICS. The Clarivate Analytics Impact Factor. 2018. Disponível em: https://clarivate.com/essays/impact-factor. Acesso em: 05 jan. 2018.

DE BELLIS, N. Bibliometrics and Citation Analysis: from the Science Citation Index to Cybermetrics. Lanham, Md: Scarecrow Press, 2009.

DECLARATION ON RESEARCH ASSESSMENT (DORA). San Francisco Declaration on Research Assessment. 2012. Disponível em: http://www.ascb.org/files/SFDe-

clarationFINAL.pdf?x30490. Acesso em: 20 jan. 2018.

EUROPEAN COMMISSION. Evaluation of research careers fully acknowledging open science practices: rewards, incentives and/or recognition for researchers practicing Open Science. Luxembourg: European Union, 2017. Disponível em:

https://ec.europa.eu/research/openscience/pdf/os rewards wgreport final.pdf. Acesso em: 20 dez. 2019. doi: 10.2777/75255.

FERNANDÉZ-LLIMÓS, F. S. Análisis de la cobertura del concepto de Pharmaceutical Care en fuentes primarias y secundarias de información. 2003. 266 f. Tese. Granada: Universidad de Granada, Departamento de Farmacia y Tecnologia Farmacéutica, 2003.

GARFIELD, E. Citation indexes for science: A new dimension in documentation through association of ideas. Science, v. 122, n. 3159, p. 108-11, 1955. Disponível em:

http://garfield.library.upenn.edu/papers/science1955.pdf. Acesso em: 10 dez. 2017.

GARFIELD, E. Citation analysis as a tool in journal evaluation. Science, v. 17, p. 471-479, 1972. Disponível em: http://www.garfield.library.upenn.edu/essays/V1p527y1962-73.pdf. Acesso em: 11 nov. 2017.

GARFIELD, E.; SHER, I. H. New Factors in the Evaluation of Scientific Literature Through Citation Indexing. American Documentation, v.4, n. 3, p. 195-201, 1963. Disponível em: http://garfield.library.upenn.edu/essays/v6p492y1983.pdf. Acesso em: 11 nov. 2017.

GUTIERRES, R. C. C.; CABRINI, G. M. C. Indicadores de avaliação de Programas de PósGraduação: um estudo comparativo na área da Matemática. Em Questão, Porto Alegre, v. 18, Edição Especial, p.81 - 97, dez. 2012. Disponível em: https://seer.ufrgs.br/EmQuestao/article/view/33192/24052. Acesso em: 9 nov. 2019.

GLÄNZEL, W.; MOED, F. H. Journal impact measures in bibliometric research. Scientometrics, Netherlands, v. 53, n. 2, p. 171-193, 2002. 
Disponível em:

https://sci2s.ugr.es/sites/default/files/files/TematicWebSites/hindex/GlanzelMoed2002.pdf. Acesso em: 20 dez. 2019. doi: 10.1023/A:1014848323806.

GRACIO, M. C.C; OLIVEIRA, E. F. T. Indicadores cientométricos normalizados: um estudo na produção científica brasileira internacional (1996 a 2011). Perspectivas em Ciência da Informação, Belo Horizonte, v.19, n.3, p.118-133, 2014. Disponível em:

http://portaldeperiodicos.eci.ufmg.br/index.php/pci/article/view/1898. Acesso em: $18 \mathrm{dez}$. 2019. doi: 10.1590/1981-5344/1898.

HICKS, D. et al. The Leiden Manifesto for research metrics. Nature, v. 520, n. 7548, p. $429-$ 431, 2015.

Disponível em: https://www.nature.com/nature/volumes/520/issues/7548. Acesso em: 18 dez. 2019. doi: 10.1038/520429a.

HIRST, G. Discipline impact factor: a method for determining core journal lists. Journal of the American Society for Information Science, v. 29, p. 171-172, 1978. doi:

10.1002/asi.4630290403.

KALTENBORN, F. K. Validity and fairness of the impact factor. German: Birkhäuser, 2004.

LEYDESDORFF, L.; BORNMANN, L. Integrated Impact Indicators (I3) compared with Impact Factors (IFs): An alternative research design with policy implications. Journal of the American Society for Information Science and Technology. v. 62, n. 11, p. 2133-2146, 2011. Disponível em:

https://pdfs.semanticscholar.org/a409/1a0f1f4d2aa2d5229b90887ab6eb63a7d0573.pdf. Acesso em: 14 fev. 2017.

MACIAS-CHAPULA, C. A. O papel da informetria e da cienciometria e sua perspectiva nacional e internacional. Ciência da Informação, Brasilia, v.27, n.2, p.134-40, maio/ago. 1998.

MAISONNEUVE, H. L'autosuffisance des dictateurs des facteurs d'impact cache leur ignorance de la bibliométrie. Médecine thérapeutique. Paris, v. 21, n. 2, p. 77-79, 2015.

doi:10.1684/met.2015.0485.

OLIVEIRA, T. M.; AMARAL, L. Políticas Públicas em Ciência e Tecnologia no Brasil: desafios e propostas para utilização de indicadores na avaliação. In: MUGNAINI, R.; FUJINO, A.; KOBASHI, N. Y. (Org.). Bibliometria e Cientometria no Brasil: infraestrutura para avaliação da pesquisa científica na Era do Big Data. São Paulo: ECA/USP, 2017. p. 189-217.

Disponível em:

http://www.livrosabertos.sibi.usp.br/portaldelivrosUSP/catalog/view/129/108/547-1. Acesso em: 20 dez. 2019. doi: 10.11606/9788572051705.

PENNER, O. et al. On the predictability of future impact in science. Scientific Reports, United Kingdom, v. 3, n. 3052, 2013. Disponível em: https://www.nature.com/articles/srep03052. Acesso em: 18 dez. 2019. doi: 10.1038/srep03052.

ROSSEAU, R. Median and percentile impact factors: a set of new indicators. Scientometrics, Netherlands, v. 63, p. 431-441, 2005. doi: 10.1007/s11192-005-0223. 
SEGLEN, P.O. Citations and journal impact factors: questionable indicators of research quality. Allergy, v. 52, v. 11, p. 1050-1056, 1997. Disponível em:

https://onlinelibrary.wiley.com/doi/epdf/10.1111/j.1398-9995.1997.tb00175.x. Acesso em: 20 dez. 2019. doi: 10.1111/j.1398-9995.1997.tb00175.x.

SIMONS, K. The misused Impact Factor. Science, v. 322, n. 5899, p. 165, 2008. doi: 10.1126/science.1165316.

SOMBATSOMPOP, N. MARKPIN, T.; PREMKAMOLNETR, N. A modified method for calculating the Impact Factors of journals in ISI Journal Citation Reports: polymer science category in 1997-2001. Scientometrics, v. 60, n. 2, p. 217-235, 2004. doi:

10.1023/B:SCIE.0000027794.98854.f6.

SPINAK, E. Indicadores cienciométricos. Brasília, Ciência da Informação, v. 27, n. 2, p. 141 148, 1998.

VAN LEEUWEN, T. Discussing some basic critique on Journal Impact Factors: revision of earlier comments. Scientometrics, v. 92, p. 443-455, 2012.

Disponível em: https://link.springer.com/content/pdf/10.1007/s11192-012-0677-x.pdf. Acesso em: 20 dez. 2019. doi: 10.1007/s11192-012-0677-x.

VAN LEEUWEN, T.; MOED H. Development and application of journal impact measures in the Dutch science system. Scientometrics, v. 53, 249; 2002. doi: 10.1023/A:1014808709694.

VARIN, C.; CATTELAN, M.; FIRTH, D. Statistical modelling of citation exchange between statistics journals. Journal Royal Statistical Society, n. 179, part 1, p. 1-63, 2016. Disponível em: https://rss.onlinelibrary.wiley.com/oi/epdf/10.1111/rssa.12124.

Acesso em 25 abr. 2018.

VINKLER, P. Possible causes or differences in information impact of journals from different subfields. Scientometrics, Netherlands, v. 20, n. 1, p. 145-161, 1991.doi: 10.1007/BF02018152.

VOGEL, M. J. M.. Uso de indicadores bibliométricos na avaliação da CAPES: o Qualis Periódicos. In: ENCONTRO NACIONAL DE PESQUISA EM CIÊNCIA DA INFORMAÇÃO, 18., 2017, Marília. ANAIS [....] Marília: PPGCI/UNESP, SP, 2017.

WALTMAN, L. A review of the literature on citation impact indicators. Journal of Informetrics, Netherlands, v.10, n. 2, p.365-391, 2016. doi: 10.1016/j.joi.2016.02.007.

WALTMAN, L.; TRAAG, V. A. Use of the journal impact factor for assessing individual articles need not be wrong. CWTS: meaningful metrics [blog], Leiden, mar. 2017. Disponível em: https://www.cwts.nl/blog?article=n-q2z254\&title=use-of-the-journal-impact-factor-forassessing-individual-articles-need-not-be-wrong\%3e.\%20Acesso. Acesso em: 20 dez. 2019.

WOUTERS P., et al. Rethink impact factors: find new ways to judge a journal. Nature, v. 569, p. 621-623, 2019. Disponível em: https://www.nature.com/articles/d41586-019-01643-3. Acesso em: 20 dez. 2019. doi: 10.1038/d41586-019-01643-3. 